\title{
A CONTINUOUS MOVEMENT VERSION OF THE BANACH-TARSKI PARADOX: A SOLUTION TO DE GROOT'S PROBLEM
}

\author{
TREVOR M. WILSON
}

\begin{abstract}
In 1924 Banach and Tarski demonstrated the existence of a paradoxical decomposition of the 3-ball $B$, i.e., a piecewise isometry from $B$ onto two copies of $B$. This article answers a question of de Groot from 1958 by showing that there is a paradoxical decomposition of $B$ in which the pieces move continuously while remaining disjoint to yield two copies of $B$. More generally, we show that if $n \geq 2$, any two bounded sets in $\boldsymbol{R}^{n}$ that are equidecomposable with proper isometries are continuously equidecomposable in this sense.
\end{abstract}

\$1. Equidecomposability and the Banach-Tarski paradox. Fix a group $G$ of isometries of $\boldsymbol{R}^{n}$ and define the following relation - which is easily seen to be an equivalence relation - on subsets of $\boldsymbol{R}^{n}$.

Definition 1.1. $A, B \subseteq \boldsymbol{R}^{n}$ are $G$-equidecomposable (written $A \sim_{G} B$ ) if there are finite partitions $\left\{A_{i}\right\}$ and $\left\{B_{i}\right\}$ of $A$ and $B$ respectively, and elements $g_{i} \in G$, so that for all $i$ we have $g_{i} A_{i}=B_{i}$. If the group $G$ is the full isometry group of $\boldsymbol{R}^{n}$, or if it is clear from context which group is intended, we will simply say that $A$ and $B$ are equidecomposable and write $A \sim B$.

Occasionally $G$-equidecomposability is called piecewise $G$-congruence. It is a much weaker condition than congruence. In particular, we have the following classical results.

Theorem 1.2 (Banach-Tarski paradox). There is a partition of the 3-ball B into two sets, each of which is equidecomposable with $B$.

Theorem 1.3 (Banach-Tarski paradox, strong form). If $n \geq 3$, then any two bounded subsets of $\boldsymbol{R}^{n}$ with nonempty interior are equidecomposable.

For modern proofs of these theorems, and other facts about equidecomposability, see, e.g., [4], [6].

§2. Continuous equidecomposability. One possible strengthening of equidecomposability is the following.

Definition 2.1. $A, B \subseteq \boldsymbol{R}^{n}$ are continuously $G$-equidecomposable (written $\left.A \approx_{G} B\right)$ if there are finite partitions $\left\{A_{i}\right\}$ and $\left\{B_{i}\right\}$ of $A$ and $B$ respectively,

Received January 20, 2005; accepted May 9, 2005.

Research supported by NSF Grant DMS 9987437. 
and a family of $G$-paths $\left\{\gamma^{i}\right\}$, so that for all $i$ we have $\gamma_{0}^{i}=e, \gamma_{1}^{i} A_{i}=B_{i}$, and $\gamma_{t}^{i} A_{i} \cap \gamma_{t}^{j} A_{j}=\emptyset$ for all $t \in[0,1]$ and all $j \neq i$. Again, if $G$ is the full isometry group, or is clear from context, we will omit it from the notation.

In contrast, the standard notion of equidecomposability in Definition 1.1-which we will call "discrete equidecomposability"-is equivalent to only requiring the $\gamma^{i}$ to be defined at $t=1$. Intuitively, in a continuous decomposition the motion can be realized physically by moving the pieces in time rather than transporting them instantaneously to their destinations.

Before proceeding we should justify our notation with the following observation.

Proposition 2.2. $\approx$ is an equivalence relation.

Proof. The trivial partition and trivial path witness $A \approx A$. If $\left\{A_{i}\right\},\left\{B_{i}\right\}$, and $\left\{\gamma^{i}\right\}$ witness $A \approx B$, then the same partitions and the paths $\left\{\gamma_{1-t}^{i}\left(\gamma_{1}^{i}\right)^{-1}\right\}$ witness $B \approx A$. If additionally $\left\{B_{j}^{\prime}\right\},\left\{C_{j}\right\}$ and $\left\{\delta^{j}\right\}$ witness $B \approx C$, then the partitions $\left\{A_{i} \cap\left(\gamma_{1}^{i}\right)^{-1} B_{j}^{\prime}\right\}_{i, j}$ and $\left\{C_{j} \cap \delta_{1}^{j} B_{i}\right\}_{i, j}$ and the paths $\left\{\gamma^{i} .\left(\delta^{j} \gamma_{1}^{i}\right)\right\}_{i, j}$ witness $A \approx C$, where "." denotes concatenation of paths.

Continuously equidecomposable sets are discretely equidecomposable, and we will shortly see that under some broad conditions the converse is true.

§3. A type semigroup for bounded sets. To avoid the explicit construction of complicated decompositions, we will develop a notion of addition of sets that allows us to combine decompositions of smaller sets into a decomposition of a larger set. We want this addition to be well-defined on continuous equidecomposability classes $(\approx$-classes.)

We will restrict our attention to the ideal $\mathscr{B}$ of bounded subsets of $\boldsymbol{R}^{n}$, which is closed under equidecomposability, and additionally require

$$
n \geq 2, \quad \boldsymbol{R}^{2} \subseteq G,
$$

where by the latter condition we mean that $G$ contains all translations in the first two dimensions - an example to keep in mind is the group of proper isometries of $\boldsymbol{R}^{n}$. These conditions have a dual purpose: to allow us to define addition of $\approx$-classes, and to show that $\approx$-classes are the same as $\sim$-classes, i.e., that continuous equidecomposability is no stronger than discrete equidecomposability for bounded sets. We will also assume the Axiom of Choice, which, along with boundedness, is required for the Banach-Tarski paradox anyway.

In the semigroup of discrete equidecomposability types (see, e.g., [4], [6],) the sum of two bounded sets may be defined as the union of disjoint translates. Since it is not a priori apparent that this operation is well-defined on continuous equidecomposability classes, we will further restrict the choice of translates. Writing $[A]$ for $\left\{A^{\prime} \in \mathscr{B}: A^{\prime} \approx A\right\}$, define addition by

$$
[A]+[B]=[(A+u) \cup(B+v)],
$$

where $u, v \in \boldsymbol{R}^{2} \subseteq G$ are chosen so that $A+u$ lies strictly to the left of $B+v$ in the first coordinate, i.e.,

$$
p_{1}(v-u)>p_{1}(a-b) \text { for all } a \in A \text { and } b \in B,
$$

where $p_{1}$ is the first coordinate projection. 
Proposition 3.1. The operation (2) is well-defined, associative, and commutative.

Proof. The sum $[A]+[B]$ is independent of the choice of $u$ and $v$ : if $u^{\prime}$ and $v^{\prime}$ also satisfy (3) then $(A+u) \cup(B+v) \approx\left(A+u^{\prime}\right) \cup\left(A+b^{\prime}\right)$ by translating $A+u$ and $B+v$ along line segments. Now we will show that $[A]+[B]$ is independent of the choices of representatives of $[A]$ and $[B]$. Suppose $A \approx A^{\prime}$ is witnessed by the pieces $\left\{A_{i}\right\}$ and $\left\{A_{i}^{\prime}\right\}$ and paths $\left\{\alpha^{i}\right\}$, and $B \approx B^{\prime}$ is witnessed by the pieces $\left\{B_{i}\right\}$ and $\left\{B_{i}^{\prime}\right\}$ and paths $\left\{\beta^{i}\right\}$. Since $A$ and $B$ are bounded and the time interval $[0,1]$ is compact we may choose $u$ and $v$ so that

$$
p_{1}(v-u)>p_{1}(a-b) \text { for all } i, j, t, a \in \alpha_{t}^{i}\left(A_{i}\right), b \in \beta_{t}^{j}\left(B_{j}\right) .
$$

Then the decomposition $[A]+[B]=\left[A^{\prime}\right]+\left[B^{\prime}\right]$ is witnessed by the pieces $\left\{A_{i}+u\right\} \cup$ $\left\{B_{j}+v\right\}$ and $\left\{A_{i}^{\prime}+u\right\} \cup\left\{B_{j}^{\prime}+v\right\}$ and the paths $\left\{\tau_{u} \alpha^{i} \tau_{-u}\right\} \cup\left\{\tau_{v} \beta^{j} \tau_{-v}\right\}$, where $\tau$ denotes translation in the first coordinate. Therefore the operation is well-defined. Associativity is trivial. For commutativity, choose $r>\operatorname{diam}(A \cup B)$; then

$$
[A]+[B]=[A \cup(B+r)]=[A \cup(B-r)]=[B]+[A]
$$

is witnessed by $\gamma^{1}=0$ and $\gamma_{t}^{2}=r e^{i \pi t}-r$, where $\boldsymbol{R}^{2} \subseteq G$ is considered as $\boldsymbol{C}$.

§4. Extricability. Next we consider a property of pairs of sets $A, B \in \mathscr{B}$ that makes the condition (3) unnecessary, so that in defining $[A]+[B]$ we can safely choose $u, v=0$ in (2).

Definition 4.1. A pair of disjoint sets $A, B \in \mathscr{B}$ is extricable if $[A]+[B]=$ $[A \cup B]$. More generally, a finite family of pairwise disjoint sets $A_{i} \in \mathscr{B}$ is extricable if $\sum_{i}\left[A_{i}\right]=\left[\bigcup_{i} A_{i}\right]$.

Intuitively, sets are extricable if they can be physically separated from each other with a finite number of cuts. Note that if $\left\{A_{i}\right\}$ is extricable and $A_{i}^{\prime} \subseteq A_{i}$ for all $i$, then the same $G$-paths extricate $\left\{A_{i}^{\prime}\right\}$. We are interested in sets that are small enough that their subsets are always extricable:

Definition 4.2. Let the class $\mathscr{E} \subseteq \mathscr{B}$ consist of bounded sets $A$ such that any two disjoint subsets of $A$ are extricable, or equivalently (by induction,) such that any finite, pairwise disjoint family of subsets of $A$ is extricable.

In fact, we will show that $\mathscr{E}=\mathscr{B}$, but first we need some lemmas.

LEMMA 4.3. $\mathscr{E}$ is closed under subsets and under unions of extricable families.

Proof. Closure under subsets is obvious. If the sets $A_{i} \in \mathscr{E}$ form an extricable family, then given finitely many pairwise disjoint $B_{j} \subseteq \bigcup_{i} A_{i}$, we have

$$
\left[\bigcup_{j} B_{j}\right]=\sum_{i}\left[\bigcup_{j}\left(A_{i} \cap B_{j}\right)\right]=\sum_{i, j}\left[A_{i} \cap B_{j}\right]=\sum_{j}\left[\bigcup_{i}\left(A_{i} \cap B_{j}\right)\right]=\sum_{j}\left[B_{j}\right]
$$

and $\left\{B_{j}\right\}$ is extricable.

LEMma 4.4. There is a partition $\left\{S_{1}, S_{2}\right\}$ of $\boldsymbol{R}$ such that the algebraic differences $\Delta S_{i}=S_{i}-S_{i}$ are codense in $\boldsymbol{R}$.

Proof. Define $K=\bigcup_{n \in N} \frac{1}{3^{n}} \boldsymbol{Z}$ and $H=K+\frac{1}{2} \boldsymbol{Z}$. Use the Axiom of Choice to select coset representatives $\left\{r_{\alpha}\right\}$ for $\boldsymbol{R} / H$, and define $S_{1}=\bigcup_{\alpha}\left(r_{\alpha}+K\right)$ and $S_{2}=S_{1}+\frac{1}{2}$. For all $a, b \in S_{i}$, if $a-b \in H$ then $a-b \in K$, so $\Delta S_{i}$ is disjoint from the dense set $H \backslash K=K+\frac{1}{2}$. 
THEOREM 4.5. $\mathscr{E}=\mathscr{R}$.

Proof. Let $A \in \mathscr{B}$. Using the sets $S_{i} \subseteq \boldsymbol{R}, i=1,2$ from above, define $S_{i j}=$ $S_{i} \times S_{j} \times \boldsymbol{R}^{n-2}$ and let $A_{i j}=A \cap S_{i j}$. Choose $r>\operatorname{diam} A$; then $\left\{A_{i j}\right\}$ is extricable by linearly translating $A_{i j}$ by $i r$ in the second dimension and then by $(2 i+j) r$ in the first, as shown schematically in Figure 1 below. Now by Lemma 4.3 it suffices to show $A_{i j} \in \mathscr{E}$. In fact, for any $i$ and $j$ there is a single path $\gamma$ in $\boldsymbol{R}^{2} \subseteq G$ that can separate any disjoint pair $B, C \subseteq A_{i j}$, defined as follows. Let $\left\{a_{k}\right\} \rightarrow 0$ and $\left\{b_{k}\right\} \rightarrow 0$ be sequences in $\boldsymbol{R} \backslash \Delta S_{i}$ and $\boldsymbol{R} \backslash \Delta S_{j}$ respectively, and define a sequence $\left\{v_{k}\right\}$ in $\boldsymbol{R}^{2} \subseteq G$ by

$$
v_{0}=\left(r, b_{0}\right), \quad v_{2 k+1}=\left(a_{k}, b_{k}\right), \quad v_{2 k+2}=\left(a_{k}, b_{k+1}\right) .
$$

Then let $\gamma$ linearly interpolate between $v_{k+1}$ and $v_{k}$ during the time interval $2^{-k-1} \leq$ $t \leq 2^{-k}$, and define $\gamma(0)=0$ (Fig. 2.) For all $t>0$ we have $\gamma_{t} \notin \Delta S_{i} \times \Delta S_{j}$, so $\gamma_{t}\left(A_{i j}\right) \cap A_{i j}=\emptyset$. Therefore $\gamma_{t}(C) \cap B=\emptyset$ for all $t$, and moreover $\gamma_{1}(C)=$ $C+\left(r, b_{0}, 0, \ldots, 0\right)$ lies strictly to the right of $B$, so $\gamma$ extricates $\{B, C\}$.

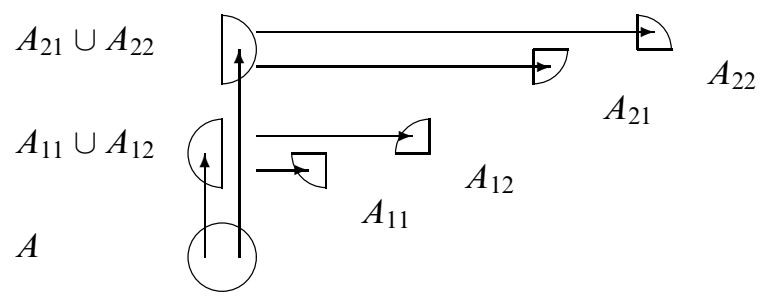

FiguRe $1 .\left\{A_{i j}\right\}$ is extricable.

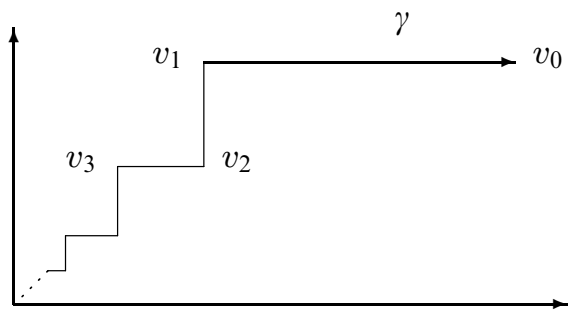

Figure 2. The path $\gamma$.

Immediately we have the following:

COROLLARY 4.6. If $n \geq 2$, any finite partition of a bounded subset of $\boldsymbol{R}^{n}$ is extricable by translations.

$\S 5$. A continuous paradox. We have shown that under the conditions (1), bounded sets may be continuously separated into pieces. If the group $G$ is path-connected, 
as is the group of proper isometries, congruences of these pieces may be realized continuously. Thus we have the following result.

THEOREM 5.1. If $n \geq 2$ and $G$ is a path-connected group of isometries of $\boldsymbol{R}^{n}$ containing all translations in two dimensions, then any two $G$-equidecomposable bounded subsets $A$ and $B$ of $\boldsymbol{R}^{n}$ are continuously $G$-equidecomposable.

Proof. Suppose that $A$ and $B$ are discretely equidecomposable using the partitions $\left\{A_{i}\right\}$ and $\left\{B_{i}\right\}$ and isometries $\left\{g_{i}\right\}$. Choosing a path from $e$ to $g_{i}$ in $G$ gives $A_{i} \approx B_{i}$, so we have $[A]=\sum_{i}\left[A_{i}\right]=\sum_{i}\left[B_{i}\right]=[B]$.

Consequently, we obtain a continuous version of the strong form of the BanachTarski paradox:

COROLlaRY 5.2. If $n \geq 3$ then any two bounded subsets of $\boldsymbol{R}^{n}$ with nonempty interior are continuously equidecomposable using proper isometries.

Proof. Simply note that the proof of the Banach-Tarski paradox (see, e.g., [4], [6],) uses only the group of proper isometries, which satisfies the conditions of the theorem.

In particular, the 3-ball has a continuous paradoxical decomposition, solving a question of de Groot posed in [1, p. 25] and again by Wagon [6, pp. 32, 232]. In the latter, the question appears in a slightly different form:

Question 5.3 (De Groot's question). Let $B, B_{1}$, and $B_{2}$ be pairwise disjoint unit balls in $\boldsymbol{R}^{3}$. Can $B$ be partitioned into sets $A_{1}, \ldots, A_{m}, A_{m+1}, \ldots, A_{m+n}$ such that for each $i=1, \ldots, m+n$ and $t \in[0,1]$ there is an isometry $\sigma_{t}^{i}$ satisfying:

1. $\sigma_{0}^{i}$ is the identity; $\sigma_{1}^{i}\left(A_{i}\right), i=1, \ldots, n$ partitions $B_{1} ; \sigma_{1}^{i}\left(A_{i}\right), i=n+1, \ldots$, $n+m$ partitions $B_{2}$.

2. For each $i$, the isometries $\sigma_{t}^{i}$ depend continuously on $t$.

3. For each $t \in[0,1]$, the sets $\sigma_{t}^{i}\left(A_{i}\right), i=1, \ldots, m+n$ are pairwise disjoint.

Corollary 5.4. De Groot's question has an affirmative answer.

Proor. By Corollary 5.2, there is a continuous decomposition $B \approx B_{1} \cup B_{2}$ witnessed by pieces $C_{i}$ and paths $\gamma^{i}$ for $i \leq n$. Set $m=n$ and define $A_{i}=$ $C_{i} \cap\left(\gamma_{1}^{i}\right)^{-1} B_{1}, A_{n+i}=C_{i} \cap\left(\gamma_{1}^{i}\right)^{-1} B_{2}$, and $\sigma^{i}=\sigma^{n+i}=\gamma^{i}$ for all $i \leq n$.

In a slightly different vein, we may apply the theorem to Laczkovich's solution [5] of Tarski's circle-squaring problem: that a circle (including interior) is equidecomposable in $\boldsymbol{R}^{2}$ with a square of equal area. In fact, Laczkovich's result uses only translations, so Theorem 5.1 immediately yields the following.

COROLlary 5.5. A circle is continuously equidecomposable by translations with a square of equal area.

§6. Necessity of conditions for theorem 5.1. In the real line, continuous equidecomposability is much stronger than discrete equidecomposabilty because it preserves order type.

Theorem 6.1. If $A, B \subseteq \boldsymbol{R}$ and $A \approx B$ by isometries, then $A$ and $B$ are orderisomorphic.

Proof. If the decomposition is witnessed by $\left\{A_{i}\right\},\left\{B_{i}\right\}$, and $\left\{\gamma^{i}\right\}$, then each $\gamma_{t}^{i}$ is in the path-component of the identity and is therefore a translation. For all $t \in[0,1]$ define the piecewise translation $g_{t}=\left.\bigcup_{i} \gamma_{t}^{i}\right|_{A_{i}}$. We have $g_{1}(A)=B$, and 
$g_{1}$ is strictly order-preserving: Fix $x, y \in A$ with $x<y$ and suppose $x \in A_{i}$ and $y \in A_{j}$. If $i=j$, then $g_{1}(x)=\gamma_{1}^{i} x<\gamma_{1}^{i} y=g_{1}(y)$. Otherwise, since $\gamma_{t}^{i} A_{i}$ and $\gamma_{t}^{j} A_{j}$ are disjoint for all $t$, the continuous function $g_{t}(x)-g_{t}(y)=\gamma_{t}^{i} x-\gamma_{t}^{j} y$ is nonzero, and since it is positive for $t=0$ it is positive for $t=1$ and $g_{1}(x)<g_{1}(y)$.

For example, $\left\{2^{-n}\right\}_{n \in N} \cup\{0\} \not \approx\left\{2^{-n}\right\}_{n \in N} \cup\{2\}$ even though the two sets are discretely equidecomposable, since only the former has a least element. The next two examples show the necessity of assuming that $G$ is path-connected, and of allowing more pieces in the decomposition.

Example 6.2. Let $n=2$ and let $G$ be the full isometry group of $\boldsymbol{R}^{2}$. Choose an infinite set $A \subseteq \boldsymbol{R}^{2}$ so that all distances in $A$ are distinct and no three points are collinear; e.g., consider $\boldsymbol{R}^{2}$ as $\boldsymbol{C}$ and let $A=\left\{e^{i \pi 2^{-n}}: n \in \boldsymbol{N}\right\}$. We claim that $A \not \approx g A$ if $g$ is an improper isometry. Otherwise, some piece in the decomposition must contain three points $\left\{a_{i}\right\}$, which map to $h a_{i}$ respectively for some isometry $h$ in the path-component of the identity. Then by uniqueness of distances we have $h a_{i}=g a_{i}$ for all $i \leq 3$. However, the images of three non-collinear points uniquely determine an isometry, so $h=g$ is improper, a contradiction.

EXAmple 6.3. Let $S$ be the unit sphere in $\boldsymbol{R}^{n}$ and choose $x \in \boldsymbol{R}^{n}$ with $|x|>3$. Then $S \cup\{x\}$ and $S \cup\{0\}$ are discretly equidecomposable by translation using the two pieces $S$ and $\{x\}$. Conversely, since $d(S, x)>\operatorname{diam}(S \cup\{0\})$, no piece of a decomposition can intersect both $S$ and $\{x\}$, so if there are only two pieces, they must be $S$ and $\{x\}$ themselves. However, if there is a continuous decomposition, then without loss of generality it fixes $S$ and connects 0 and $x$ with a path in $\boldsymbol{R}^{n} \backslash S$, which is impossible.

\section{$\S 7$. Remarks.}

Remark 7.1. A re-examination of Section 4 shows that Corollary 4.6 may be strengthened to place a bound on the number of pieces. In extricating an $n$ partition of a bounded set $A$, the only subdivision occurs when we take a common refinement with the partition $\left\{A_{i j}=A \cap S_{i j}\right\}_{i, j=1,2}$ in Theorem 4.5, so each of the original pieces is divided in four, and the partition may be extricated in $4 n$ pieces. Likewise, Theorem 5.1 can be strengthened to say that if the bounded sets $A$ and $B$ are equidecomposable in $n$ pieces, then they are continuously equidecomposable in $16 n$ pieces: each piece is divided in four to be extricated from $A$, and its image under the decomposition is divided in four to be extricated from $B$. In fact, we can do slightly better: without loss of generality one piece of the decomposition remains fixed, so these two subdivisions coincide and it is divided in four only once. Therefore $A$ and $B$ are continuously equidecomposable in $4^{2}(n-1)+4=16 n-12$ pieces.

In the special case of paradoxical decompositions of a ball we can do better still. There is a discrete decomposition of a ball $B$ with two copies $B_{1} \cup B_{2}$ using five pieces, described in [6, p. 41]. By translating and rotating $B_{1}$ and $B_{2}$, we may assume that each of them contains a piece whose total movement is a translation by some element of the subgroup $K^{\prime}=K \times K \times \boldsymbol{R}^{n-2}$ of $\boldsymbol{R}^{n}$, with $K$ from Lemma 4.4; then since the sets $S_{i j}$ are invariant under $K^{\prime}$, these two pieces are only subject to one subdivision each. Moreover, another one of the pieces is a single point, which 
cannot be subdivided at all. Thus a continuous decomposition may be accomplished in $4^{2} \cdot 2+4 \cdot 2+1=41$ pieces.

Remark 7.2. Dougherty and Foreman [2] give a version of the Banach-Tarski paradox using sets with the Baire property. However, the sets $S_{i}$ in Lemma 4.4 cannot have the Baire property, since otherwise one of them would be nonmeager and by Pettis's Theorem (see, e.g., [3]) its algebraic difference $\Delta S_{i}$ would contain a neighborhood of zero. Therefore our procedure for generating a continuous decomposition from a discrete one does not preserve the Baire property in the pieces, and the question of the existence of a continuous paradox using pieces with the Baire property is left open.

§8. Acknowledgments. This paper is the result of an undergraduate research project supported by an NSF grant. The author would like to thank Prof. A. S. Kechris of Caltech for his time and guidance.

\section{REFERENCES}

[1] Theodorus Jozef Dekker, Paradoxical decompositions of sets and spaces, Ph.D. thesis, Drukkerij Wed. G. van Soest, Amsterdam, 1958.

[2] Randall Dougherty and Matthew Foreman, Banach-Tarski paradox using pieces with the property of Baire, Proceedings of the National Academy of Sciences of the United States of America, vol. 89 (1992), no. 22, pp. 10726-10728.

[3] Alexander S. Kechris, Classical descriptive set theory, Graduate Texts in Mathematics, vol. 156, Springer-Verlag, New York, 1995.

[4] MikLós LaCZKOVICH, Equidecomposability and discrepancy; a solution of Tarski's circle-squaring problem, Journal für die Reine und Angewandte Mathematik, vol. 404 (1990), pp. 77-117.

[5] - Equidecomposability of sets, invariant measures, and paradoxes, Rendiconti dell'Istituto di Matematica dell'Università di Trieste, vol. 23 (1991), no. 1, pp. 145-176, (1993), School on Measure Theory and Real Analysis (Grado, 1991).

[6] Stan Wagon, The Banach-Tarski paradox, Encyclopedia of Mathematics and its Applications, vol. 24, Cambridge University Press, Cambridge, 1985.

DEPARTMENT OF MATHEMATICS

CALIFORNIA INSTITUTE OF TECHNOLOGY

PASADENA, CA 91125, USA

E-mail: trev@its.caltech.edu 